\title{
A PERCEPÇÃO DAS FAMÍlIAS SOBRE A ORGANIZAÇÃO DA ATENÇÃO PRIMÁRIA À SAÚDE DA CRIANÇA
}

\author{
Ana Paula Pereira Fernandes ${ }^{1}$, Ana Maria Cosvoski Alexandre ${ }^{1}$, Ana Paula Dezoti², \\ Verônica de Azevedo Mazza ${ }^{3}$
}

\begin{abstract}
RESUMO: Pesquisa descritiva, qualitativa com o objetivo de compreender, a partir da percepção das famílias, a organização e as práticas de cuidado à criança na atenção primária à saúde; foi desenvolvida com 45 representantes de famílias de crianças de 0 a 5 anos, inscritas nos programas de três unidades de saúde de um município da região metropolitana de Curitiba- Paraná. Os dados foram coletados de setembro de 2008 a fevereiro de 2009, por meio de três grupos focais e analisados segundo análise temática categorial. Emergiram três categorias empíricas: Acesso aos serviços de saúde; Integralidade; e Acolhimento. Na percepção das famílias há questões favoráveis em relação à reformulação das práticas dos programas para a saúde da criança. Entretanto, referem barreiras de acesso decorrentes de questões de organização dos serviços para a população fora destes programas. Portanto, considera-se imprescindível a participação das famílias no planejamento das ações de atenção à saúde da criança.
\end{abstract}

DESCRITORES: Enfermagem; Saúde da criança; Família; Atenção primária à saúde.

\section{LA PERCEPCIÓN DE LAS FAMILIAS ACERCA DE LA ORGANIZACIÓN DE LA ATENCIÓN PRIMARIA A LA SALUD DEL NIÑO}

RESUMEN: Investigación descriptiva, cualitativa con el objetivo de comprender, con base en la percepción de las familias, la organización y las prácticas de cuidado al niño en la atención primaria a la salud. Fue desarrollada con 45 representantes de familias de niños de 0 a 5 años, inscriptas en los programas de tres unidades de salud de un municipio de la región metropolitana de Curitiba - Paraná. Los datos fueron obtenidos de septiembre de 2008 a febrero de 2009, por medio de tres grupos focales y analizados según análisis temático de categoría. Resultaron tres categorías empíricas: Acceso a los servicios de salud; Integralidad; y Acogimiento. En la percepción de las familias hay cuestiones favorables acerca de la reformulación de las prácticas de los programas para la salud del niño. Sin embargo, hay obstáculos de acceso a causa de cuestiones de organización de los servicios para la población fuera de estos programas. Por lo tanto, se considera imprescindible la participación de las familias en el planeamiento de las acciones de atención a la salud del niño.

DESCRITORES: Enfermería; Salud del niño; Familia; Atención primaria a la salud.

\section{FAMILIES' PERCEPTIONS REGARDING THE ORGANIZATION OF PRIMARY HEALTH CARE FOR THEIR CHILDREN}

ABSTRACT: This descriptive, qualitative research aimed to understand, based on the families' perception, the organization and the practices of care for the child in primary health care; the study was undertaken with 45 representatives of families of children aged from zero to five years old, enrolled in the programs of three health centers in a municipality in the metropolitan region of Curitiba, in the state of Paraná. The data was collected between September 2008 and February 2009, through three focus groups, and was analyzed using categorical thematic analysis. Three empirical categories emerged: Access to the health services; Integrality; and Embracement. In the perception of the families, there are issues which are favorable in relation to the reformulation of the practices of the programs for child health. However, they mention barriers to access, resulting from issues of organization of the services for the population not included in these programs. It is therefore considered essential for the families to participate in the planning of the health care actions for the child.

DESCRIPTORS: Nursing; Child health; Family; Primary health care.

${ }^{1}$ Enfermeira. Mestre em Enfermagem. Universidade Federal do Paraná. Curitiba-PR-Brasil

${ }^{2}$ Enfermeira. Mestranda em Enfermagem. Universidade Federal do Paraná. Professora da Universidade Estadual do Centro-Oeste. Guarapuava-PR-Brasil

${ }^{3}$ Enfermeira. Doutora em Enfermagem.Professora da Universidade Federal do Paraná. Curitiba-PR-Brasil

Autor correspondente:

Recebido: $27 / 11 / 2012$

Ana Paula Pereira Fernandes.

Aprovado: 18/12/2013

Universidade Federal do Paraná

Rua Ailton Luiz Nodari, 121 - 83407-730 - Colombo-PR-Brasil

E-mail: anaenfermagem1@yahoo.com.br

Cogitare Enferm. 2014 Jan/Mar; 19(1):56-62 


\section{INTRODUÇÃO}

O Sistema Único de Saúde vem sendo reestruturado para atender às reais necessidades de saúde da população e, como exemplos de estratégias inovadoras, apontam-se a Atenção Primária e a Estratégia Saúde da Família. Com isto, torna-se indispensável repensar o direcionamento da produção dos serviços em saúde, em sua lógica de abordar somente as manifestações patológicas, o que a caracteriza como fragmentadora, curativista e pontual.

É preciso trabalhar sob a ótica integral, isto é, refletir as práticas de saúde a partir da leitura ampliada da realidade de vida dos indivíduos, garantindo que as intervenções se deem em consonância com as singularidades de cada um deles ${ }^{(1)}$.

Faz-se necessário entender a forma de organização dos serviços a partir da dialética entre as demandas de saúde atendidas e as necessidades percebidas pelas famílias, para o fortalecimento de projetos de intervenções relacionados ao desenvolvimento infantil ${ }^{(2)}$, pois a família produz cuidados essenciais à saúde de seus membros ${ }^{(3)}$. Para tanto, os usuários têm o direito de participar das decisões, mesmo sendo verificado que este processo participativo é deixado de lado no momento da formação dos serviços ${ }^{(4)}$.

Portanto, o objetivo deste estudo foi compreender, a partir da percepção das famílias, a organização e as práticas de cuidado à criança na atenção primária à saúde.

\section{MÉTODO}

Trata-se de pesquisa do tipo descritiva com abordagem qualitativa, realizada em um município da região metropolitana de Curitiba. A rede de atenção primária à saúde nesta região é composta de 21 Unidades de Saúde (US), sendo 8 Unidades com Estratégia Saúde da Família (UESF) e 13 Unidades Básicas de Saúde (UBS). Foram selecionadas três UESF, sugeridas pelos gestores, tendo como critério maior demanda de atendimento infantil.

Foram convidados a participar da pesquisa todos os membros de 45 famílias residentes nas áreas de abrangência das unidades, sendo 15 famílias de cada US. No entanto, apenas 15 mães e duas avós de diferentes famílias e áreas de abrangência aceitaram participar do estudo. O critério de inclusão foi famílias com crianças de zero a cinco anos inscritas em algum dos programas da US.

Os dados foram coletados entre os meses de se- tembro de 2008 a fevereiro de 2009, por meio de três seções de grupos focais, com roteiro semiestruturado que abordou as facilidades e dificuldades em relação ao atendimento das crianças nas UBS, e sobre quais melhorias as famílias apontam para estes serviços.

Os depoimentos foram gravados e transcritos na íntegra, sendo nomeados nos discursos com GF (grupo focal) seguidos da sequência numérica em que ocorreram (GF1, GF2 e GF3). Para análise dos dados utilizou-se a análise temática categorial ${ }^{(5)}$, que pressupõe as etapas de pré-análise, exploração do material ou codificação, tratamento dos resultados, inferência e interpretação.

O projeto seguiu a Resolução 196/96 do Conselho Nacional de Saúde. Obteve-se a concordância dos gestores de saúde do município e aprovação do Comitê de Ética e Pesquisa do Setor de Ciências da Saúde da Universidade Federal do Paraná, sob registro CEP/SD: 376.056.07.07.

\section{RESULTADOS}

A partir da análise temática as seguintes categorias e subcategorias são apresentadas.

\section{Acesso ao serviço de saúde}

Subcategoria - Acesso ao serviço de saúde pelos programas de saúde da criança

O acesso ao serviço, representado pelos programas de saúde à criança, foi considerado satisfatório. Foram citados: a puericultura, a imunização e o programa do leite, permitindo às crianças prioridade de inserção nestes programas e demais serviços da US.

O programa de imunização foi indicado pelas famílias, apontado como pontos positivos os mecanismos de acesso como a flexibilização no horário de atendimento e a disponibilidade dos tipos de vacinas para a infância, como descrito na fala a seguir:

A vacina, eu faço aqui [...]. Sempre tem as que precisam, em qualquer horário. (GF 2)

Outro programa referido como facilitador para o cuidado infantil na organização dos serviços de saúde da criança foi o programa do leite. Todavia, as famílias mencionaram sua operacionalização insatisfatória quanto ao dia e horário propostos, que limitam e conflitam com a pesagem da criança e a entrega do leite. Foi sugerida a necessidade de revisão desta prática, como referido a seguir: 
Aqui, é um dia a cada 2 meses [pesagem do programa do leite], e vira um tumulto. Tem um monte de mães esperando, e às vezes não dá tempo de buscar o leite, que é no mesmo dia até às 9 horas. Só que para você pegar o leite lá, tem que pesar. (GF 3)

A fala de GF3 explicita que a organização do serviço deve ser repensada para atender às necessidades de saúde. Para tanto, as famílias pontuaram que é preciso um espaço para escuta entre usuários e trabalhadores da saúde e gestores, sobre as dificuldades enfrentadas na utilização do serviço, tal como relatado a seguir:

Se a gente fizesse uma reunião entre todo mundo aqui que tem uma coisa para reclamar sobre o posto de saúde, como a gente está fazendo aqui? Aqui estão só os pais, mas se a gente fizesse isso com alguém da prefeitura? Olha, a gente precisa de um pediatra, de mais remédio; a gente está precisando disso, daquilo. (GF 3)

Subcategoria - Acesso ao serviço de saúde pela demanda espontânea

As famílias citaram a demanda espontânea como um dos meios de se conseguir o atendimento. Contudo, este acesso geralmente é referido para crianças com faixa etária maior de dois anos, que estão excluídas do programa de puericultura do município e permeado por descrições de ineficácia, pois não consegue atender as necessidades dos usuários que buscam pelo serviço, de acordo com as falas a seguir:

Às vezes tem dez consultas; a gente vem, fica um tempão e não consegue vaga! (GF 1)

[...] tem que ficar pagando consulta, lá no " $X$ ”, [clínica particular]. Por causa disso, se o nenê fica doente [...] estou pagando para ser melhor atendida. (GF 3).

Deve-se reforçar que no município existe o pré-agendamento de consultas médicas e a integração com profissionais, como o dentista e o nutricionista, apenas para as crianças que estão inseridas nos programas de saúde da criança, remetendo as demais crianças aos critérios da demanda espontânea.

As falas de GF1 e GF3 expressam as dificuldades de acesso a estes serviços de saúde, tais como filas madrugueiras, falta de vagas para consultas e incompatibilidade de horário de funcionamento das US com a jornada de trabalho dos pais. Tais dificuldades são referidas como situações que contribuem para a inversão na lógica do modelo de atenção idealizado de entrada no sistema pela atenção primária, e a proximidade do território, espaço da vida como lócus da atenção à saúde, para o distanciamento do serviço de referência de sua área de residência, em busca de serviços para responder às suas necessidades de saúde.

\section{A Integralidade dos serviços de saúde}

Quando o foco é o sistema para integrar os serviços dos níveis primário, secundário e terciário, em especial no que se refere à referência e contrarreferência do município, várias dificuldades foram percebidas pelas famílias, as quais se refletem em riscos para a saúde das crianças, além do descrédito destes mecanismos, com falhas no controle de regulação de vagas, com longas filas de espera, como na fala a seguir.

O meu filho teve um problema do coração em 2001. Eu trouxe ele aqui com um papel para encaminhar. Tem um mês que o papel chegou. Meu filho já está com 7 anos, e faz menos de 1 mês que o papel chegou [...]. Eu levei no particular, senão meu filho ia morrer [...]. Fez exames e detectou veia entupida no coração. Tivemos que fazer uma cirurgia de emergência. (GF 4)

\section{Acolhimento}

O acolhimento, preconizado pelo SUS, foi reconhecido e referido pelas famílias como uma prática de qualidade, restrita a alguns profissionais que a utilizam como ferramenta em sua atuação, tal como por um familiar:

O Dr. X era um ótimo médico aqui, mas ele foi embora [...]. Ele pesquisa o que a criança tem, não pede exame, examina, ouve a gente, tem paciência na consulta. (GF 3)

As propostas de acolhimento devem ser articuladas com as famílias, que já sugeriram nos grupos focais algumas medidas, tais como o pré-agendamento das consultas, a reserva de vagas, a flexibilidade do horário de atendimento, e até mesmo abordagem mais atenciosa, paciente e de corresponsabilização, como se percebe em discurso a seguir:

[...] agendar hoje para semana que vem... Nem que 
demorasse uma ou duas semanas, mas que não tivesse que madrugar na fila! (GF 1)

\section{DISCUSSÃO}

Os programas de saúde que são adotados pelos municípios são originários de estratégias clínico-epidemiológicas visando reduzir os índices de agravo na população. Apesar das dificuldades experimentadas, os municípios são responsáveis pela realização de intervenções específicas - as ações de prevenção e promoção da saúde - de forma descentralizada ${ }^{(6)}$. Esta descentralização permite a organização diferenciada dos serviços de saúde de modo a atender às reais necessidades da população.

A delimitação na faixa etária de atendimento no programa de puericultura acaba gerando exclusão das crianças que não se encontram nesta faixa etária. Reflete-se que o acesso à atenção primária para essas famílias pode ser considerado focalizado, mas este restringe $o$ atendimento a determinados serviços ou programas, figurando a universalização da atenção em um sistema segmentado e desarticulado na cadeia organizacional do sistema público como um todo ${ }^{(7)}$.

Institui-se um desafio aos gestores e trabalhadores locais: a expansão ou criação de medidas de maior acesso para a faixa etária de crianças que estão fora deste programa. Indica-se que as práticas de acompanhamento à criança nestas UBS podem ainda estar focadas no modelo centrado no médico, na consulta médica, porque as famílias não citaram práticas assistenciais desempenhadas por outros profissionais na US visando ao Desenvolvimento Infantil. Há de se explorar as habilidades da equipe ESF a fim de se produzir uma cultura de promoção da saúde, prevenção de agravos, tratamento e reabilitação.

O programa de imunização, referido pelas famílias, tem como objetivo a administração e acompanhamento da situação vacinal infantil, trabalhando-se com tipos de vacinas padronizadas pelo município a partir de necessidades de controle epidemiológico. A credibilidade do Programa de Imunização pode ser devido a sua efetividade, da gratuidade deste serviço, bem como resultado de investimento do governo com programas e metas específicas para a imunização, incluindo as frequentes campanhas de divulgação junto à população e aos trabalhadores de saúde ${ }^{(8)}$.

O referido programa do leite, citado por seu importante acesso ao atendimento em saúde, foi implantado em vários municípios do Paraná, com o objetivo de combate à desnutrição, por meio da distribuição de um litro de leite diário à família de crianças entre seis meses e três anos, com renda per capita de no máximo meio salário mínimo ${ }^{(9)}$. Por meio deste, as unidades de saúde realizam o acompanhamento no crescimento e desenvolvimento destas crianças, realizando a medição e pesagem.

A participação das famílias na concepção e operacionalização das práticas dos serviços de saúde é fundamental e ocorre no município do estudo por meio do Conselho Municipal de Saúde. Todavia, estas anseiam pela participação no planejamento local das unidades, e que os gestores estivessem cientes destas discussões para garantir as mudanças que lhes competem. Assinala-se que espaços de escuta nas unidades de saúde devem ser estabelecidos de forma organizada e frequente, assim como as avaliações das ações e atividades de saúde para estas famílias.

O planejamento local dos serviços configura-se um projeto coletivo para integrar cuidados de saúde, no qual trabalhadores, gestores e usuários são corresponsáveis no fazer saúde ${ }^{(7)}$.

Para as mudanças na organização do serviço, também é preciso compromisso dos governos em estabelecer políticas públicas de proteção e promoção à saúde da criança, e assim contribuir para diminuir as desigualdades que condicionam o processo saúde-doença. Portanto, o grau de investimento que o Estado disponibiliza para o financiamento das ações de atendimento infantil está relacionado com o acesso aos serviços de saúde e à qualidade de vida da população ${ }^{(10)}$.

$\mathrm{O}$ acesso ao serviço de saúde, no que se refere à demanda espontânea, é permeado pelas dificuldades referidas, somando-se a uma cultura biologicista e curativista, e a um número limitado de vagas para consulta médica ofertadas por dia nas US, concentradas no período da manhã, gerando filas madrugueiras, e ainda determinadas por ordem de chegada ao serviço. Tal forma de acesso não deixa claro, para os entrevistados, se existe uma lógica de prioridades a ser atendida pelos serviços de saúde.

Assim, ofertar medidas de acesso aos serviços de saúde com qualidade para a população significa minimizar ou quebrar barreiras que permeiam a obtenção do cuidado, que podem ser referentes à organização dos serviços de saúde e ao processo de trabalho das instituições, extrapolando aspectos geográficos, socioeconômicos e culturais ${ }^{(11)}$.

Na premissa de que saúde é um direito universal, cabe ao estado a função de oferecer à população condi- 
ções de acesso aos serviços de saúde para um exercício pleno de vida, compreendendo o processo de saúde e doença e suas causas socioeconômicas ${ }^{(12)}$.

A reorganização do serviço de saúde e das práticas profissionais torna-se mais complexa quando a relação entre a oferta e demanda não é bem conhecida, fazendo com que as ações desenvolvidas aconteçam de acordo com as demandas nos serviços ${ }^{(13)}$. A necessidade de priorizar grupos para a ação, como a puericultura, pode originar outro grupo de usuários de saúde insatisfeitos por não obterem os mesmos benefícios, considerando os serviços não resolutivos e buscando equipamentos que não possuem características de atenção primária. Esta prática demonstra que os usuários constroem seu próprio itinerário para sanar os problemas de saúde-doença, com base nas crenças, conhecimento e facilidades que encontram nos serviços de saúde ${ }^{(11)}$. Percebe-se uma inversão da lógica da porta de entrada, que deveria ser pelas US, podendo provocar descontinuidade no cuidado do usuário.

A fim de responder às necessidades de cuidado à criança, as famílias precisam contar com uma rede hierarquizada de cuidados com profissionais e serviços dentro e fora das próprias US e até mesmo articulando serviços que ultrapassam as delimitações municipais. Nesta vertente, a concepção da integralidade está ligada à organização da atenção primária e a sua responsabilidade em prestar assistência contínua à população, pois consiste na porta de entrada do usuário aos níveis de atenção, propiciando a interpretação dos problemas dentro do contexto histórico-social do usuário ${ }^{(14)}$. Para promover cuidados integrados é preciso estruturas organizacionais compartilhando ideias com arranjos de rede, gestão de processos e gestão da doença ${ }^{(15)}$.

Compreende-se que a fila pela atenção ao nível secundário e terciário de saúde também é um problema de outras cidades, como foi citado em um estudo realizado em Fortaleza que apontou que nesta fila de espera os usuários podem permanecer por um ano ou mais. Entre as causas dessa dificuldade, os autores do referido estudo citam a falta de articulação entre o nível de atenção primária de saúde e os demais níveis do sistema, fazendo com que alguns usuários se obriguem a pagar consultas com especialistas ${ }^{(16)}$.

É válido lembrar a responsabilidade da US em relação à contrarreferência e acompanhamento dos casos. É preciso organizar o atendimento de acordo com as necessidades e gravidade do indivíduo. É apropriado agir de forma equitativa, ou seja, a ordem de atendimento e procedimento deve ser equivalente aos danos à saúde, evitando maiores comprometimentos à saúde das pessoas.
A não articulação entre o serviço de referência e contrarreferência acarreta uma não comunicação entre os serviços de saúde em seus diferentes níveis de atenção e, por sua vez, possíveis prejuízos à continuidade da assistência ${ }^{(17)}$. Portanto, na organização dos serviços de saúde deve objetivar-se a integralidade na estruturação dos serviços, com adequado funcionamento do sistema de referência/contra-referência, uma vez que a resolução dos problemas de saúde muitas vezes implica nos devidos encaminhamentos para serviços de saúde especializados $^{(17)}$.

Retoma-se aqui a integralidade dos serviços, para atender aos diferentes níveis de atenção, percebendo a necessidade em reorganizar as instituições e suas práticas, de modo a promover acesso a uma rede hierarquizada de cuidados integrais e com profissionais cujo foco esteja voltado para um cuidado que compreenda o indivíduo em todas as suas dimensões, inclusive considerando as prioridades necessárias em cada realidade.

Acesso e acolhimento são complementares na implementação das práticas nos serviços de saúde em busca de um atendimento integral ${ }^{(11)}$. Ao refletir sobre a estruturação do SUS como oferta de serviços, que possui limites para o atendimento da livre demanda, criando redes hierarquizadas e prioritárias, as medidas de acolhimento tornam-se essenciais na relação cotidiana entre usuários e serviços de saúde.

Compreende-se o acolhimento por duas vertentes: uma pautada na postura dos profissionais de saúde para atender e suprir as expectativas dos usuários, intermediando as dimensões das ofertas e possibilidades do serviço com as necessidades expressas pelos usuários. A outra, compreendida pela organização do serviço de saúde como mecanismo planejado e implementado para reorganizar o trabalho e a estrutura da US para atender à demanda espontânea e organizada nos programas, melhorando as condições de acesso ao serviço como um todo ${ }^{(18)}$. Destaca-se que ambas as perspectivas são eficazes e complementares para resolução dos problemas de saúde da população.

Para qualificar o cuidado, deve-se apostar na construção conjunta dos modos de produzir saúde dentro das instituições. Neste sentido, a organização do serviço tem influência desta construção que deve ter como base as reais necessidades de quem procura o serviço aliado às informações epidemiológicas e ao uso do território como lócus das práticas de saúde. Assim, deve-se contar com a incorporação de novas estratégias de qualificação e ampliação das ações, como: projeto terapêutico singular, clínica ampliada e apoio matricial ${ }^{(19)}$. 
As atividades rotineiras desempenhadas na atenção primária quando associadas às técnicas de acolhimento podem suscitar adesão maior do usuário, assim como ampliar o vínculo usuário-profissional/serviço de saúde, fundamental no efetivo acompanhamento da saúde de crianças na faixa etária de zero aos cinco anos e no fortalecimento de suas famílias.

Discutir o acolhimento e o acesso à atenção à saúde ultrapassa a dimensão singular, requer reflexões da forma como a equipe de saúde organiza o atendimento à população, e a compreensão de como o Estado assume o compromisso com a saúde da população expressa nas políticas de saúde, financiamento e na manutenção e fortalecimento de espaços democráticos de construção coletiva para dar voz e reconhecimento às necessidades de saúde da população.

Ressalta-se que o processo de trabalho destes profissionais deve ser conhecido, assim como as possibilidades na sua formação e capacitação para melhor compreender questões que podem influenciar na relação usuários-profissionais. A pressão exercida em uma demanda espontânea desorganizada pode ser um destes problemas, e faz pensar que os profissionais de saúde também precisam de uma acolhida em seu processo de trabalho, com a devida capacitação, incentivos, escuta, autonomia e estratégias de cuidado ao cuidador ${ }^{(11)}$.

\section{CONSIDERAÇÕES FINAIS}

As famílias apontaram pontos favoráveis e negativos com relação à organização da atenção primária. Compreende-se que existem necessidades de reestruturação das práticas e organizações dos serviços de saúde que perpassam as dimensões locais das US, pois estão impregnadas por questões sócio-históricas da atenção primária, e expressam competências políticas e estruturais das esferas municipal, estadual e federal.

No entanto, percebe-se o potencial do acolhimento como uma ferramenta valiosa para minimizar as insatisfações das famílias na organização dos serviços, especialmente flexibilizando as questões de acesso ao serviço e assim promovendo o vínculo entre a US e a comunidade, e abrindo espaços para diálogo a fim de captar as reais necessidades das famílias para o cuidado da criança, em busca do empoderamento de ambas as partes. Deste modo, considera-se imprescindível a participação das famílias no planejamento das ações de atenção primária para a promoção da saúde das crianças.

O desenvolvimento de outros estudos pode apontar para reflexões enovas experiências acerca do processo de trabalho dos profissionais de saúde em diferentes realidades e para as possibilidades de interconectar as necessidades de saúde das famílias para o cuidado da criança.

\section{REFERÊNCIAS}

1. Bonfada D, Cavalcante JRLP, Araujo DP, Guimarães J. A integralidade da atenção à saúde como eixo da organização tecnologia nos serviços. Ciênc. saúde colet. [Internet] 2012;17(2) [acesso em 14 ago 2013]. Disponível: http://www.redalyc.org/pdf/630/63020718028.pdf

2. Ferreira JC, Fernandes APP, Souza C, Bicudo DO, Mazza VA. A percepção do gestor sobre a organização da atenção básica à saúde da criança. Cogitare enferm. [Internet] 2010;15(1) [acesso em 12 set 2013]. Disponível: http://ojs.c3sl.ufpr.br/ojs2/index.php/ cogitare/article/view/17140/11283

3. Gutierrez DMD, Minayo MCS. Produção de conhecimento sobre cuidados da saúde no âmbito da família. Ciênc. saúde colet. [Internet] 2010;15(Suppl 1) [acesso em 14 ago 2013]. Disponível: http://dx.doi. org/10.1590/S1413-81232010000700062

4. Carlos EF, Silva CC, Silva ATMC, Braga JEF. Programa de Saúde da Família: inclusão dos usuários na escolha dos serviços oferecidos. Rev. brasciSaúde. [Internet] 2009;13(2) [acesso em 12 ago 2013]. Disponível: http://periodicos. ufpb.br/ojs2/index.php/rbcs/article/v view/3266/4299

5. Bardin L. Análise de conteúdo. Lisboa: Edições 70; 1977.

6. Paulus Junior A, Gualda NLP, Cordoni Junior L. Efeitos da descentralização da saúde no município de Londrina. Rev. Espaço Saúde. [Internet] 2011;13(1) [acesso em 16 set 2013]. Disponível: http://www.uel.br/revistas/uel/ index.php/espacoparasaude/article/view/ 9530/pdf

7. Assis MMA, Nascimento MAA, Franco TB, Jorge MSB (orgs). Produção do cuidado no Programa Saúde da Família: olhares analisadores em diferentes cenários [Internet]. Salvador: EDUFBA, 2010. [acesso em 6 dez 2013]. Disponível: http://dx.doi. org/10.7476/9788523208776

8. Lopes EG, Martins Christine BG, Lima FCA, Gaíva MAM. Situação vacinal de recém-nascidos de risco e dificuldades vivenciadas pelas mães. Rev. bras. enferm. [Internet] 2013;66(3) [acesso em 8 nov 2013]. Disponível: http://dx.doi.org/10.1590/S0034-71672013000300006

9. Brasil. Lei Estadual n. 16.475, de 22 de abril de 2010. Instituiu o ProgramaLeite das Crianças no Paraná. Sistema 
Estadual de Legislação, 2013. [acesso em 10 dez 2013] Disponível: http://www.legislacao.pr.gov.br/legislacao/ pesquisarAto.do?action $=$ exibir\&cod Ato $=55028$.

10. Silva DI, Chiesa AM, Veríssimo MDR, Mazza VA. Vulnerabilidade da criança diante de situações adversas ao seu desenvolvimento: proposta de matriz analítica. Rev Esc Enferm USP. No prelo 2014.

11. Souza ECF, Vilar RLA, Rocha NSPD, Uchoa AC, Rocha PM. Acesso e acolhimento na atenção básica: uma análise da percepção dos usuários e profissionais de saúde. Cad. Saúde Pública. [Internet] 2008;24(Suppl 1) [acesso em 18 nov 2013]. Disponível: http://dx.doi. org/10.1590/S0102-311X2008001300015

12. Mattos RA. Princípios do Sistema Único de Saúde (SUS) e a humanização das práticas de saúde. Interface (Botucatu). [Internet] 2009;13 (Suppl 1) [aceso em 18 nov 2013]. Disponível: http://dx.doi.org/10.1590/S141432832009000500028

13. Costa MCG, et al. As ações dos serviços de saúde voltadas para o âmbito individual e pouco coletivo. Rev. bras. educ. med. [Internet] 2012;36 (Suppl 1) [acesso em 18 nov 2013]. Disponível: http://dx.doi.org/10.1590/ S0100-55022012000200008

14. Starfield B. Atenção primária: equilíbrio entre necessidades de saúde, serviços e tecnologia. Brasília: UNESCO/Ministério da Saúde; 2004.

15. Kodner D. Together Now: A Conceptual Exploration of Integrated Care. Healthcare Quarterly. [Internet] 2009;13(n. esp.) [acesso em 22 nov 2013]. Disponível: http://www.longwoods.com/content/21091

16. Coelho MO, Jorge MSB, Araujo ME. O acesso por meio do acolhimentonaatenção básicaà saúde. Rev. Baiana. [Internet] 2009;3(3) [acesso em 22 nov 2013]. Disponível: http:// files.bvs.br/upload/S/0100-0233/2009/v33n3/a011.pdf

17. Escorel S, Giovanella L, Mendonça MHM, Senna MCM. O Programa de Saúde da Família e a construção de um novo modelo para a atenção básica no Brasil. Rev. Panam. Salud Públ. [Internet] 2007;21(2-3) [acesso em 28 nov 2013]. Disponível: http://dx.doi.org/10.1590/ S1020-49892007000200011

18. Takemoto MLS, Silva EM. Acolhimento e transformações no processo de trabalho de enfermagem em unidades básicas de saúde de Campinas, São Paulo, Brasil. Cad. Saúde Pública. [Internet] 2007;23(2) [acesso em 28 nov 2013]. Disponível: http://dx.doi. org/10.1590/S0102-311X2007000200009
19. Souza CR, Botazzo C. Construção social da demanda em saúde. Physis [Internet] 2013;23(2) [acesso em 28 nov 2013]. Disponível: http://dx.doi.org/10.1590/S010373312013000200005

Cogitare Enferm. 2014 Jan/Mar; 19(1):56-62 